\title{
A pulsatile hemodynamic evaluation of the commercially available bifurcated Y-graft Fontan modification and comparison with the lateral tunnel and extracardiac conduits
}

\author{
Phillip M. Trusty, BS, ${ }^{\mathrm{a}}$ Maria Restrepo, PhD, ${ }^{\mathrm{a}}$ Kirk R. Kanter, MD, ${ }^{\mathrm{b}}$ Ajit P. Yoganathan, PhD, ${ }^{\mathrm{a}}$ \\ Mark A. Fogel, MD, ${ }^{\mathrm{c}}$ and Timothy C. Slesnick, $\mathrm{MD}^{\mathrm{d}}$
}

\begin{abstract}
Objective: Fontan completion, resulting in a total cavopulmonary connection, is accomplished using a lateral tunnel, extracardiac conduit, or bifurcated Y-graft. The use of Y-grafts is hypothesized to provide symmetric hepatic blood flow distribution to the lungs, a factor related to pulmonary arteriovenous malformations. The present study evaluates the hemodynamic performance of the largest commercially available Y-graft cohort to date, highlights 6 representative cases, and compares commercially available Y-graft performance with lateral tunnel/extracardiac conduit connections.
\end{abstract}

Methods: A total of 30 patients with commercially available Y-grafts and 30 patients with lateral tunnel/extracardiac conduits were analyzed. Total cavopulmonary connection anatomies and flow waveforms were reconstructed using cardiac magnetic resonance images and phase-contrast cardiac magnetic resonance. Computational fluid dynamic simulations were performed to quantify total cavopulmonary connection power loss, resistance, and hepatic flow distribution. Comparisons between graft types were investigated.

Results: Total cavopulmonary connection resistance was significantly higher for Y-grafts. Hepatic flow distribution was similar overall but showed discrepancies at extreme values with more unbalanced flow in the Y-graft cohort. Power loss was more sensitive to left pulmonary artery stenosis in the Y-graft cohort. Prediction of Y-graft hepatic flow distribution is multifactorial.

Conclusions: Commercially available Y-grafts do not inherently provide more balanced hepatic flow distribution than lateral tunnel/extracardiac conduit connections, which are more energetically favorable and less sensitive to pulmonary artery stenosis. Graft type should be considered on an individual basis because hemodynamic performance is based on a combination of factors, including pulmonary flow distribution, pulmonary artery stenosis, and superior vena cava positioning. (J Thorac Cardiovasc Surg 2016;151:1529-36)



Fontan Y-graft HFD; streamlines colored by inlet vessel.

\section{Central Message}

Commercially available Y-grafts do not inherently provide more balanced HFD or favorable energetic performance than traditional Fontan connections.

Perspective

Despite intuition, commercially available Ygrafts do not inherently provide more balanced HFD than traditional Fontan connections. Several Y-grafts were seen to direct nearly all hepatic flow to a single lung. Graft type should be considered on an individual basis because hemodynamic performance is governed by several factors, including PFD and PA stenosis.

See Editorial Commentary page 1537.
The Fontan procedure is the final operation in a series of palliative surgical procedures for patients with single ventricle congenital heart defects, in which the inferior

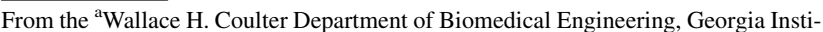
tute of Technology and Emory University; 'bivision of Cardiothoracic Surgery, and ${ }^{\mathrm{d}}$ Division of Cardiology, Department of Pediatrics, Children's Healthcare of Atlanta, Emory University School of Medicine, Atlanta, Ga; and ${ }^{\mathrm{c} D i v i s i o n}$ of Cardiology, Children's Hospital of Philadelphia, Philadelphia, Pa.

Received for publication Dec 16, 2015; revisions received March 1, 2016; accepted for publication March 5, 2016; available ahead of print April 5, 2016.

Address for reprints: Ajit P. Yoganathan, PhD, Wallace H. Coulter Department of Biomedical Engineering, Georgia Institute of Technology and Emory University, Atlanta, GA 30332-0535 (E-mail: ajit.yoganathan@bme.gatech.edu). $0022-5223 / \$ 36.00$

Copyright (c) 2016 by The American Association for Thoracic Surgery http://dx.doi.org/10.1016/j.jtcvs.2016.03.019
}

vena cava (IVC) is connected to the pulmonary arteries (PAs) by way of an extracardiac conduit (ECC) or intracardiac tunnel. This operation results in the total cavopulmonary connection (TCPC) that bypasses the ventricle with passive venous return into the PAs. ${ }^{1}$ With advances in medical imaging, clinicians can better determine the appropriate course of action for individual patients

Scanning this QR code will take you to the article title page.

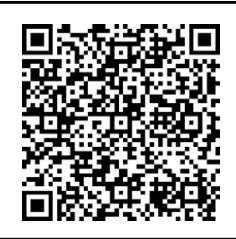




$$
\begin{aligned}
& \text { Abbreviations and Acronyms } \\
& \text { BSA = body surface area } \\
& \text { CFD }=\text { computational fluid dynamics } \\
& \text { ECC }=\text { extracardiac conduit } \\
& \text { HFD }=\text { hepatic flow distribution } \\
& \text { iPL }=\text { indexed power loss } \\
& \text { IVC }=\text { inferior vena cava } \\
& \text { LPA }=\text { left pulmonary artery } \\
& \text { LT }=\text { lateral tunnel } \\
& \text { PA }=\text { pulmonary artery } \\
& \text { PAVM }=\text { pulmonary arteriovenous malformation } \\
& \text { PFD }=\text { pulmonary flow distribution } \\
& \text { PI }=\text { pulsatility index } \\
& \text { PL }=\text { power loss } \\
& \text { RPA }=\text { right pulmonary artery } \\
& \text { SVC }=\text { superior vena cava } \\
& \text { TCPC }=\text { total cavopulmonary connection } \\
& \text { WU }=\text { Wood units }
\end{aligned}
$$

before surgery. Previous studies focused on surgical planning for the Fontan operation in an attempt to maximize energy efficiency, obtain equal hepatic flow distribution (HFD), and potentially improve the patient's long-term quality of life. ${ }^{2-4}$ Further efforts characterized the effect of baffle diameter, vessel offset, connection angle, and surgical approach on the power loss (PL) and HFD of the TCPC. ${ }^{5-10}$

Currently, surgeons complete the Fontan connection using a lateral tunnel (LT) baffle, ECC, or Y-graft. The ECC is typically positioned externally around the margin of the heart, and the LT is positioned through the atrium. LT/ ECC connections usually are anastomosed to the mid-PA, inferior to the superior vena cava (SVC) anastomosis from the prior bidirectional Glenn. Alternatively, the commercially available bifurcated Y-graft modification splits inferior systemic venous return through 2 branches toward the left and right pulmonary arteries. Y-grafts are used in an effort to obtain balanced HFD to the left and right lungs, a factor in preventing pulmonary arteriovenous malformations (PAVMs), a potential Fontan patient morbidity. ${ }^{11,12}$ The TCPCs from 2 patients using each option are shown in Figure 1.

For the inherently inefficient circulation in patients undergoing the Fontan, all attempts to minimize the ventricle's workload are clinically valuable. Surgeons can repair branch pulmonary artery (PA) stenosis, determine energetically favorable baffle placement, and choose which connection type to use. However, surgeons may not have complete freedom in these choices because they are limited by the specific patient's anatomy and condition.
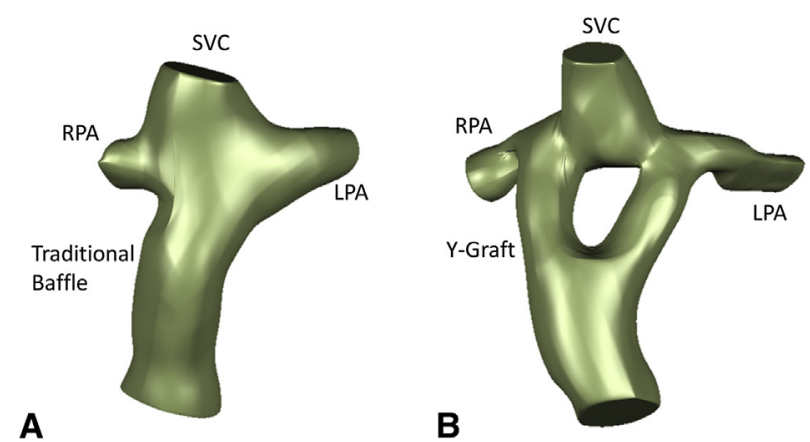

FIGURE 1. A, TCPC with an extracardiac baffle. B, TCPC with a Y-graft. $R P A$, Right pulmonary artery; $S V C$, superior vena cava; $L P A$, left pulmonary artery.

The potential energetic and HFD differences associated with graft choice have not been fully analyzed in large cohorts under pulsatile simulation conditions. Previous studies showed exploratory and preliminary results discussing the effectiveness of Y-graft use, but included very small sample sizes. ${ }^{13-18}$ Some of these studies used time-averaged boundary conditions, which can cause non-negligible differences in TCPC hemodynamics. ${ }^{9,19}$ The present study compares hemodynamic performance between LT/ECC and commercially available Y-graft TCPCs with the largest patient cohort to date while using patient-specific transient flow boundary conditions to provide insights into graft choice for Fontan completion.

\section{MATERIALS AND METHODS Patient Cohort}

A total of 60 patients were used in this study. All LT/ECC patient data $(\mathrm{n}=30)$ were received from Children's Healthcare of Philadelphia, and all Y-graft patient data $(\mathrm{n}=30)$ were received from Children's Healthcare of Atlanta. Thirty consecutive Y-grafts performed by Dr Kirk Kanter were used; patients undergoing LT/ECC were chosen to match age and body surface area (BSA) between groups. All Y-graft Fontan connections used commercially available Y-grafts. Clinical data including age, gender, and BSA were obtained for each patient. The Institutional Review Boards of the institutions involved approved this study.

\section{Anatomy and Velocity Reconstruction}

Patient-specific anatomies were reconstructed from axial cardiac magnetic resonance images using methods previously developed. ${ }^{20,21}$ Geomagic Studio (Geomagic Inc, Research Triangle Park, NC) was then used to fit a surface around the reconstructed point-cloud and export the surface for mesh generation. Patient-specific blood flow waveforms were calculated from phase-contrast magnetic resonance images for all vessels of interest using previously validated methods. ${ }^{22,23}$

\section{Computational Fluid Dynamics}

All geometries were imported into GAMBIT (ANSYS Inc, Lebanon, $\mathrm{NH})$ for mesh generation. To provide computational stability, flow extensions of $10 \mathrm{~mm}$ and $50 \mathrm{~mm}$ were added to all inlets and outlets, respectively. All computational fluid dynamic (CFD) simulations were completed using a previously developed and validated in-house immersed boundary solver. ${ }^{4,5,14,24}$ The unsteady flow waveforms extracted from phase-contrast magnetic resonance imaging were imposed as boundary 
conditions for each inlet and outlet. A total of 6 cardiac cycles were simulated for each patient to overcome transition effects and achieve periodic stability; the final cycle was used for hemodynamic analysis.

\section{Data Analysis}

Calculations were performed to compute PL, indexed power loss (iPL), TCPC resistance, pulsatility index (PI), and percent stenosis. PL was calculated as follows:

$$
P L=\sum_{\text {inlets }} \int_{A}\left(P+\frac{1}{2} \rho v^{2}\right) v \cdot d A-\sum_{\text {outlets }} \int_{A}\left(P+\frac{1}{2} \rho v^{2}\right) v \cdot d A
$$

where $p$ is the static pressure, $\rho$ is the blood density, $A$ is the area of the inlet/ outlet, and $v$ the velocity. PL was calculated for 200 points throughout the cardiac cycle and then averaged to obtain an overall PL value. PL was indexed (iPL) as $\left(\frac{P L}{\rho Q_{s}^{3} / B S A^{2}}\right)$, and a TCPC resistance was calculated $\left(\frac{\triangle P_{T C P C}}{Q_{s}}\right)$ where $Q_{s}$ is the systemic venous flow $(\mathrm{L} / \mathrm{min}), \mathrm{BSA}$ is the BSA $\left(\mathrm{m}^{2}\right), \Delta P_{T C P C}$ is the pressure decrease, and $\rho$ is the blood density $\left(\mathrm{kg} \cdot \mathrm{m}^{-3}\right)$. PI was defined as follows:

$$
P I=\frac{Q_{\max }-Q_{\min }}{2 * Q_{\text {avg }}}
$$

where $Q_{\text {max }}, Q_{\text {min }}$, and $Q_{a v g}$ are the maximum, minimum, and average flow rates during the cardiac cycle, respectively, as determined from the phase-contrast cardiac magnetic resonance data. Percent stenosis was calculated for the pulmonary arteries using the following equation:

$$
\text { Stenosis } \%=100\left(\frac{A_{\min , L P A}+A_{\min , R P A}}{A_{\text {avg }, L P A}+A_{\text {avg, }, R P A}}\right)
$$

where $A_{\min , ~}{ }_{P A}$ and $A_{\min , R P A}$ are the minimum cross-sectional areas of the left pulmonary artery (LPA) and right pulmonary artery (RPA), and $A_{\text {avg, } L P A}$ and $A_{\text {avg, } R P A}$ are the average cross-sectional areas of the LPA and RPA. A combined outlet stenosis value was used in place of unilateral PA stenosis measurements to give a more accurate representation of the total outlet obstruction to flow and allow a better comparison between patients. In addition, IVCs and SVCs showed negligible percent stenosis and therefore were not included in this calculation. All vessel areas for this metric were calculated using Vascular Modeling Toolkit version 1.0.1 (Orobix, Bergamo, Italy).

HFD was evaluated using particle tracking. Particles were seeded at the base of the LT/ECC or Y-graft at 200 time points throughout the cardiac cycle. An additional 4 cardiac cycles (with no particle seeding) were simulated to ensure that the residence time of the particles would not exceed the simulation time. Particles exiting at each outlet were counted, and HFD was defined as the ratio of particles leaving the LPA:

$$
H F D=\frac{\text { Particles }_{L P A}}{\text { Particles }_{L P A}+\text { Particles }_{R P A}}
$$

HFD will be discussed in terms of "HFD deviation from 50\%," and an even (50/50) split of hepatic flow to the LPA and RPA is assumed as ideal. This convention gives a " 0 " HFD deviation for balanced HFD cases and an HFD deviation of 50 for extreme cases (all hepatic flow to the LPA or RPA). Particle residence time was calculated by recording the amount of time required for each particle to exit the TCPC domain. An overall pulmonary flow distribution (PFD) was calculated as the ratio of LPA flow to total pulmonary flow:

$$
P F D=\frac{Q_{L P A}}{Q_{\text {Total Pulmonary }}}
$$

\section{Statistics}

The Anderson-Darling test was used to determine normality for each parameter within both patient groups. Depending on normality, a Wilcoxon rank-sum test (non-normal distribution) or a 2-sample $t$ test (normal distribution) was used to test for equal medians between patient groups using SPSS (IBM Corp, Version 23, Armonk, NY). An asterisk (*) is used to signify statistical significance using $\alpha=0.05$. Values are shown as mean \pm standard deviation.

\section{RESULTS}

The commercially available Y-graft evaluation includes an investigation of several hemodynamic metrics within the Y-graft cohort, then an examination of both ideally and poorly performing Y-grafts (in terms of HFD), and finally a comparison between Y-graft and LT/ECC performance.

\section{Y-Graft Hemodynamic Performance}

Figure 2 shows several clinically important hemodynamic metrics for the Y-graft cohort. The average PFD and HFD deviations from an ideal 50/50 split were $13.4 \pm 10.6$ and $25.9 \pm 15.1$, respectively (Figure 2, $A$ ). Figure 2, $B$ illustrates the effect of LPA stenosis on iPL. A moderate positive correlation $(r=0.67, P<.01)$ was found between LPA stenosis and iPL. The distributions of TCPC resistance and HFD deviation are shown in Figure 2, $C$ and $D$. The distribution of TCPC resistance is skewed to the right with approximately $53 \%$ and $70 \%$ of Y-graft cases having resistances less than 1 and 2 Wood units (WU), respectively. HFD deviation was relatively evenly distributed (Figure 2, D). Only 13\% of Y-graft cases have HFD deviations less than 10 (ideal), and $24 \%$ of Y-graft cases have deviations greater than 40 (poor).

\section{Representative Y-Graft Cases}

Figure 3 shows streamlines for 6 representative cases from the Y-graft cohort of 30 patients. Patients Y1, Y2, and $\mathrm{Y} 3$ show balanced HFD (low HFD deviation), whereas the final 3 (Y4, Y5, and Y6) are unbalanced. Y1 represents an "ideal" case with balanced PFD, normal SVC positioning, and no mid-PA stenosis. Y2 represents uneven PFD with mid-PA stenosis on the low PFD side. Y3 has bilateral SVCs, balanced PFD, and mid-PA stenosis. Y4 has balanced PFD with the SVC positioned superior to a Y-branch anastomosis. Y5 has unbalanced PFD with mid-PA stenosis on the high PFD side, and Y6 represents balanced PFD with Y-branch stenosis. HFD to the LPA is noted for each model in Figure 3.

\section{Y-Graft Versus Lateral Tunnel/Extracardiac Conduit}

To further evaluate the commercially available Y-graft, a direct hemodynamic comparison was performed between the Y-graft and LT/ECC cohorts. Clinical data are shown in Table 1 for each graft type. Patients were selected to best match age and BSA between groups. Y-graft resistance was significantly higher $(P=.03)$ than LT/ECC resistance, 


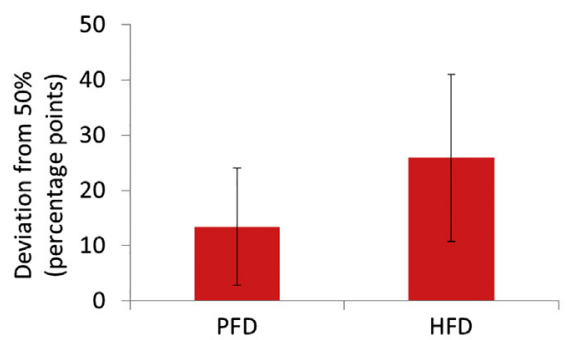

A

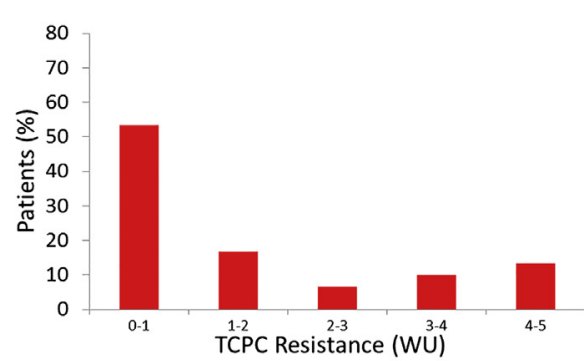

C

FIGURE 2. Y-graft hemodynamic performance. A, PFD and HFD deviations. B, Effect of LPA stenosis on iPL. C, Distribution of TCPC resistance. D, Distribution of HFD deviation. PFD, Pulmonary flow distribution; HFD, hepatic flow distribution; $L P A$, left pulmonary artery; TCPC, total cavopulmonary connection; $W U$, wood units.

with averages of $1.51 \pm 1.52 \mathrm{WU}$ and $0.56 \pm 0.54 \mathrm{WU}$, respectively (Figure 4, A). Overall, HFD deviation was not significantly different between the groups with an average of $21 \pm 13$ for the LT/ECC and $26 \pm 15$ for the Y-grafts (Figure 4, B). Figure 4, $C$ shows no significant differences in particle residence time.

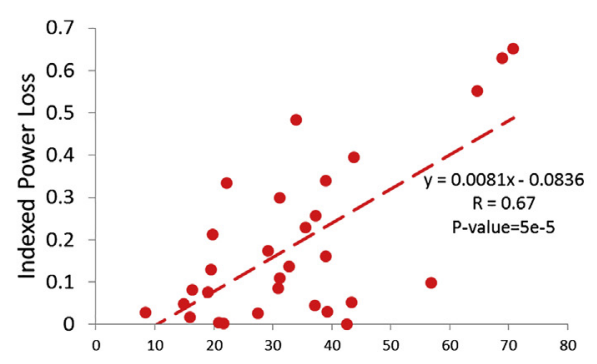

B

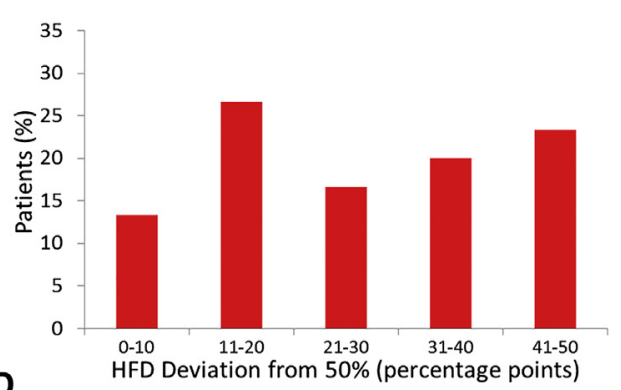

Figure 4, $D$ and $E$ compare the distribution of TCPC resistance and HFD deviation between graft types. Approximately $77 \%$ of LT/ECC cases have a resistance less than $1 \mathrm{WU}$, compared with $53 \%$ of the Y-graft cases. In addition, $30 \%$ of commercially available Y-graft cases exhibit a resistance greater than $2 \mathrm{WU}$, whereas only $3 \%$
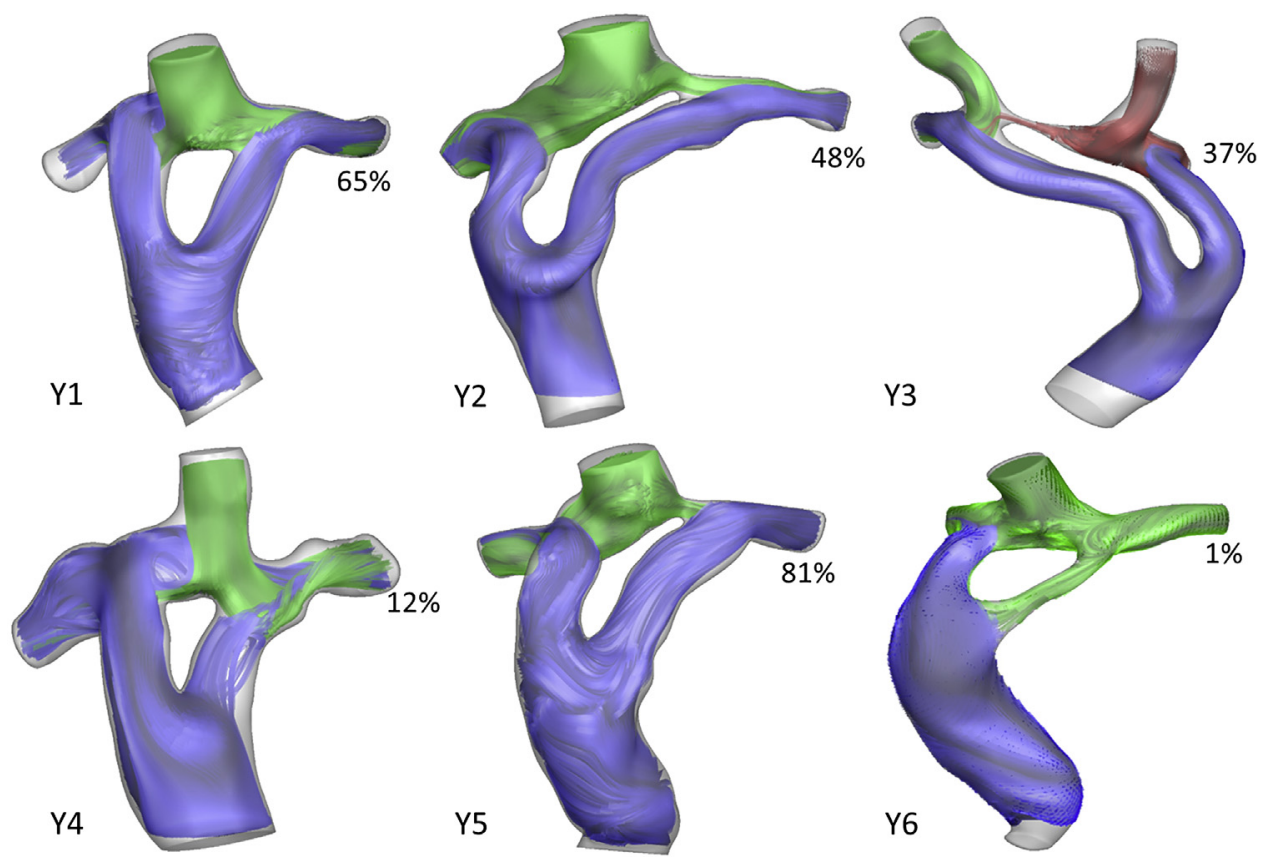

FIGURE 3. Representative Y-graft cases with HFD noted. Streamlines colored by origin: IVC (blue), SVC (green), and LSVC (red). Percentages shown are percent of hepatic flow to the LPA. 
TABLE 1. Averaged clinical data for all patients separated by graft type

\begin{tabular}{lccc}
\hline & $\begin{array}{c}\text { LT/ECC } \\
(\mathbf{n}=\mathbf{3 0})\end{array}$ & $\begin{array}{c}\text { Y-graft } \\
(\mathbf{n}=\mathbf{3 0})\end{array}$ & $\boldsymbol{P}$ value \\
\hline BSA $\left(\mathrm{m}^{2}\right)$ & $0.67 \pm 0.12$ & $0.66 \pm 0.20$ & .19 \\
Age (y) & $3.65 \pm 1.14$ & $3.68 \pm 2.49$ & .17 \\
Gender (male) & $70 \%$ & $53 \%$ & .19 \\
PA stenosis (\%)* & $33.99 \pm 12.60$ & $26.82 \pm 10.69$ & .02 \\
Systemic venous flow (L/min)* & $2.07 \pm 0.61$ & $1.57 \pm 0.59$ & $<.01$ \\
PFD (\% LPA) & $46.01 \pm 15.00$ & $46.00 \pm 16.78$ & .99 \\
PFD deviation from 50\% & $13.13 \pm 7.91$ & $13.40 \pm 10.60$ & .78 \\
$\quad$ & & & \\
$\quad$ percentage points) & $0.66 \pm 0.65$ & $0.93 \pm 0.72$ & .14 \\
PI (IVC) & $0.51 \pm 0.50$ & $0.42 \pm 0.12$ & .76 \\
\hline
\end{tabular}

Systemic venous return is defined as the sum of all TCPC inlet flow rates. PFD is the percent of total inlet flow that exits through the LPA. $L T$, Lateral tunnel; $E C C$, extracardiac conduit; $B S A$, body surface area; $P A$, pulmonary artery; $P F D$, pulmonary flow distribution; $L P A$, left pulmonary artery; $P I$, pulsatility index; $I V C$, inferior vena cava; $S V C$, superior vena cava. *Represents statistically significant differences.

of the LT/ECC cohort have values this high (Figure 4, D). By focusing on the 2 extremes of HFD deviation, $30 \%$ of LT/ECC cases had ideal HFD deviations $(<10)$ compared with only $10 \%$ of Y-graft cases. At the other extreme, more than twice as many Y-graft cases $(23 \%)$ have HFD deviations greater than 40 (poor) than LT/ECC cases $(10 \%)$.

Figure $4, F$ compares the sensitivity of iPL to LPA stenosis for both graft types. Commercially available Y-grafts were found to be more sensitive to LPA stenosis in terms of iPL with a significant difference in the 2 correlations (Fisher exact test, 1 -tailed, $z=-1.76, P=.04$ ).

\section{DISCUSSION}

By capitalizing on the relatively large sample size in this study, the following section evaluates commercially available Y-graft performance and generalizes differences between graft types. Both clinical and physiologic implications are discussed. Six Y-grafts that illustrate common anatomic parameter combinations will be emphasized, and study limitations will be considered.

\section{Y-Graft Hemodynamic Performance}

As expected, PFD deviation is relatively low, and on average there is an approximately $25 \%$ difference in overall flow rates to the LPA and RPA. This flow split is determined partially by branch PA stenosis. ${ }^{25}$ HFD deviation was higher and more variable than expected and will be discussed in the following section. The effect of LPA stenosis on iPL also is an expected result. Blood accelerates through the stenosis region and then decelerates as the vessel returns to its original size according to the conservation of momentum principle. These changes in velocity result in an increased pressure decrease across the TCPC (lower efficiency), which leads to an increased iPL. The LPA is a common site for stenosis, and Figure 2, $B$ shows that the most severe LPA stenosis anatomies have the highest iPL. ${ }^{10}$

The distribution of TCPC resistance raises concern for a number of patients with Y-grafts. In this study, Y-graft TCPCs are seen to have resistances as high as 4.7 WUs, and this increased level of vascular resistance could lead to venous hypertension/congestion. Liver failure and protein-losing enteropathy are thought to be direct results of increased Fontan venous pressures. ${ }^{26-28}$ The relatively even distribution of HFD deviation suggests that the Y-graft is not a "one size fits all" solution to accomplish evenly split hepatic flow to the lungs. Although $13 \%$ of patients with Y-grafts had very balanced HFD, approximately one quarter of patients had more than $90 \%$ hepatic flow directed to the left or right lung. The effectiveness of the Y-graft use seems to be multifactorial and extremely patient-specific, suggesting the need for preoperative, individual surgical planning via computational modeling.

\section{Representative Y-Graft Cases}

The ability of a Y-graft to evenly split hepatic flow is highly dependent on PFD, the position of branch PA stenosis, and SVC flow collision, among other factors. A difference in PFD can cause 2 Y-grafts with similar anatomies to have drastically different HFDs. This can be seen in Figure 3, in which Y2 and Y5 have PFDs of 24\% and $65 \%$, respectively. Despite similar anatomies and Y-grafts, the HFD for these cases is considerably different ( $48 \%$ and $81 \%$ to the LPA, respectively). A comparison between $\mathrm{Y} 1$ and $\mathrm{Y} 4$ illustrates the effect of SVC flow collision on HFD. Both cases have balanced PFD and similar sized PAs, but Y1 has a medially positioned SVC, whereas Y4's SVC is positioned more superior to the left Y-branch. The SVC flow collision in Y4 forces some SVC flow into the left Y-branch, which causes hepatic flow to preferentially course through the right Y-branch. The result is a very unbalanced HFD for Y4 (12\% to the LPA) and relatively balanced HFD for Y1 ( $65 \%$ to the LPA).

Stenosis is commonly seen in Fontan anatomies ${ }^{10}$; Y2, Y3, Y5, and Y6 all have severe stenosis in the TCPC. For single SVC anatomies (Y2, Y5), mid-PA stenosis influences SVC flow, which will preferentially follow the path of least resistance (in these cases toward the RPA). For an unbalanced PFD case, if the mid-PA stenosis is located on the same side as the PA receiving the majority of pulmonary flow, then the stenosis blocks most SVC flow and this PA receives the majority of its flow from the hepatics and therefore will have an unbalanced HFD (Y5). However, the opposite is true if the stenosis is located on the low PFD side; in this case hepatic flow is able to move toward both PAs resulting in more balanced HFD (Y2). For 

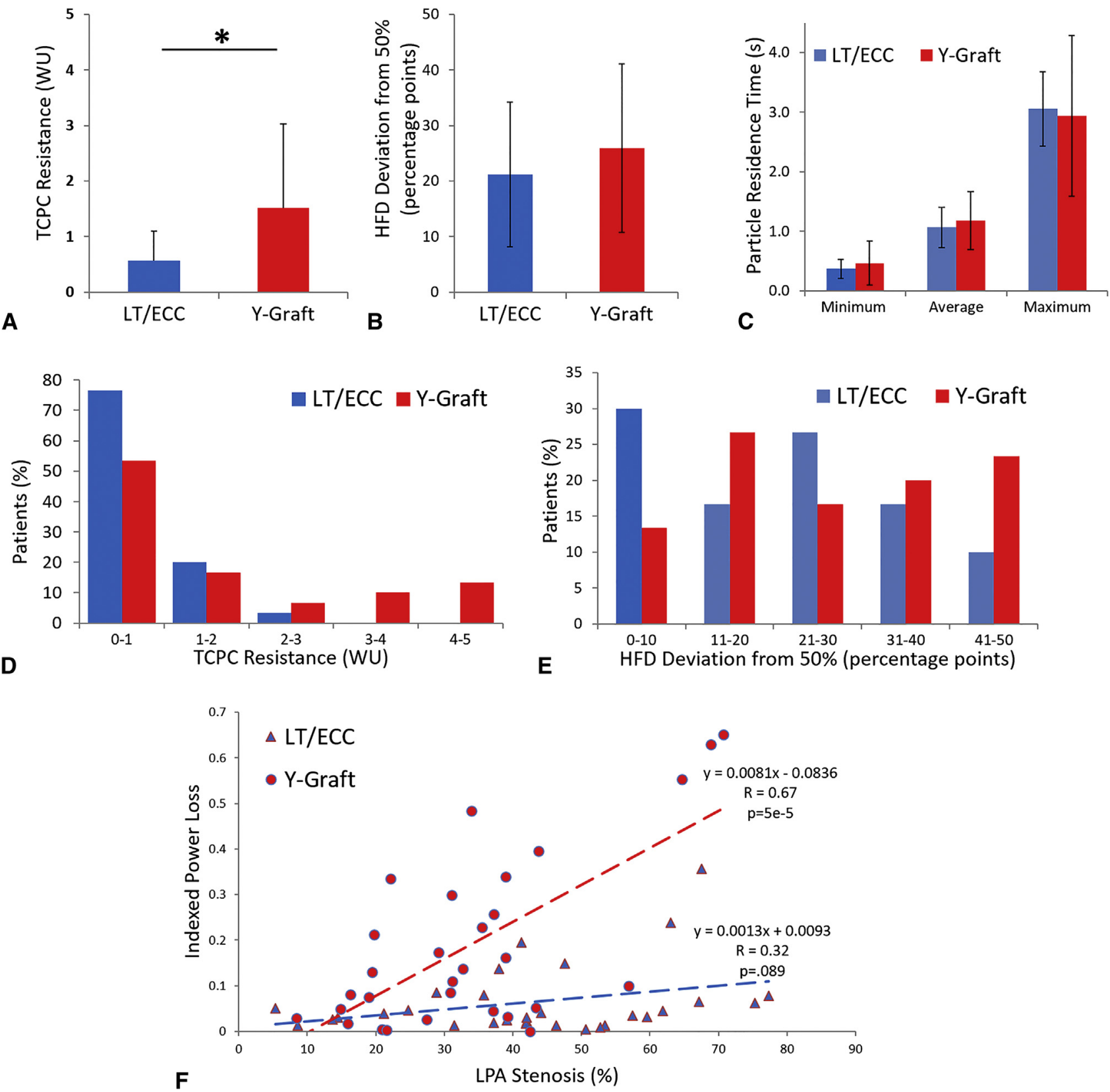

FIGURE 4. Hemodynamic comparison of the Y-graft and LT/ECC. A, iPL. B, HFD deviation from 50\%. C, Particle residence time. D, Distribution of iPL. E, Distribution of HFD deviation. F, Effect of LPA stenosis on iPL. TCPC, Total cavopulmonary connection; $W U$, wood units; $L T$, lateral tunnel; $E C C$, extracardiac conduit; $H F D$, hepatic flow distribution; $L P A$, left pulmonary artery. *Statistical significance $(P<.05)$.

bilateral SVC anatomies (Y3), mid-PA stenosis can "separate" the 2 SVC flows and HFD will be highly dependent on the individual SVC and overall PA flows. If SVC flows are similar and PFD is balanced, a balanced HFD is expected. Y6 shows a case with severe stenosis located in the left Y-branch, and as a result hepatic flow is limited through the stenosed branch. With the full range of flow rates, flow ratios, pulsatilities, unique geometries, and stenosis locations, the ability of a specific Y-graft to achieve balanced HFD may not be intuitive. Again, Y-graft performance needs to be evaluated on an individual basis, and all of these factors must be considered.

\section{Y-Graft Versus Lateral Tunnel/Extracardiac \\ Conduit}

Average TCPC resistance was approximately 3 times higher for Y-grafts than for LT/ECCs. The most apparent explanation stems from the geometry of the Y-graft itself. 
With commercially available Y-grafts, the diameter of the base of the Y-graft is evenly split between the 2 branches. For example, a 20-mm (diameter) Y-graft will have two 10 -mm (diameter) branches. A basic calculation reveals that preserving diameter does not preserve area; this design results in a 50\% reduction in cross-sectional area as blood moves into the Y-graft branches. In essence, Y-grafts have a built-in $50 \%$ long segment "stenosis." Resistance is inversely proportional to the fourth power of the radius (Poiseuille's law). Therefore, blood traveling through 2 Y-branches with diameters half the size of the LT/ECC would analytically yield an 8 times increase in resistance. According to the results presented, the reduction in diameter and cross-sectional area inherent to commercially available Y-grafts has a greater negative impact on TCPC resistance than the venous flow collisions inherent to LT/ECCs. Therefore, "custom" Y-grafts that preserve cross-sectional area as shown by Yang and colleagues ${ }^{15,18}$ may be the more promising option for successful Y-graft use and may provide an advantage over the LT/ECC Fontan pathway.

Overall, HFD deviation was not significantly different between graft types. Both graft types were highly variable. The most important observation is the discrepancy in the number of patients at both the lowest (ideal) and highest (poor) HFD deviation (Figure 4, E). In this study, Y-grafts have approximately twice as many poor differential cases as ideal cases, whereas LT/ECCs show the opposite trend. These results highlight the need for preoperative planning with Y-graft use and the design of individualized Y-grafts, which are tailored for a specific patient. Potential modifications such as the angle of origin, branch size, and overall shape could lead to better HFD performance on an individual basis.

Particle residence time was investigated for its potential impact on flow-induced thrombus formation, as well as to provide quantitative information about flow stagnation. ${ }^{29,30}$ No differences were seen for minimum, mean, or maximum residence times. As shown in Figure 4, F, Y-graft iPL was more sensitive to LPA stenosis than LT/ECC iPL. PA stenosis may have a greater influence on the reduced flow through the closest Y-branch than it does on the total hepatic flow traveling through an LT/ECC connection. This difference would lead to a greater effect of stenosis on iPL for Y-grafts than LT/ECCs.

Several additional comparisons between graft types are important to note. First, PFD was identical between graft types at $46 \%$ to the left lung, indicating that graft type does not affect PFD. In addition, there was significantly more PA stenosis $(P=.02)$ in the LT/ECC cohort, as shown in Table 1. Even with significantly more PA stenosis, which is known to increase resistance, the LT/ECC cohort still had lower TCPC resistance than the Y-grafts. This strengthens the finding that commercially available Y-grafts inherently create higher TCPC resistance. Finally, the LT/ECC group includes both intra-atrial and extracardiac connections, whereas Y-grafts are always extracardiac. In this study, 10 of the LT/ECCs were intra-atrial. To ensure that these results were a consequence of graft type and not the intra-atrial versus extracardiac variation, a subcomparison was performed between these 2 groups. No statistically significant differences were found in this subcomparison for iPL, PFD, or HFD, and therefore the comparative results presented are due to graft choice and not its location.

\section{Study Limitations}

The period of time between the Fontan operation and the magnetic resonance imaging scan was not identical for all patients included in this study. Patients receiving a Y-graft were scanned within 1 month of the surgery, and patients receiving $\mathrm{LT} / \mathrm{ECC}$ were scanned at approximately 6 months after the operation. This difference in recovery time may lead to variations in the amount of physiologic adaptation, which could affect flow rates (as seen in the difference between systemic venous flows) and pulmonary vascular resistance, and therefore TCPC resistance and HFD. Also, the spatial velocity profiles used at the inlets of the CFD domain are assumed to be parabolic, which are not identical to the spatial velocity profiles obtained from the magnetic resonance imaging velocity segmentations. However, this assumption is expected to have only minimal effects on the bulk hemodynamic metrics. From a modeling perspective, this study focused only on the TCPC geometry and did not include interactions with the rest of the cardiovascular or pulmonary circulation. This localized TCPC modeling may omit significant global influences on the system. Finally, because of limited data, the hepatic veins were not included in this modeling. The inclusion of the hepatic veins and their respective flow rates could influence the mixing of hepatic factor with the IVC flow and therefore alter the presented HFD results. This study also assumes rigid walls in the CFD simulations.

\section{CONCLUSIONS}

In this study, commercially available Y-grafts were found to have highly variable HFD due to a combination of factors, including PFD to the left and right lungs, presence and location of stenosis, and SVC positioning. HFD distributions suggest that LT/ECCs are more likely to avoid extremely unbalanced hepatic flow to the lungs. Y-graft HFD performance is multifactorial, which requires patient-specific computational modeling. Y-grafts were found to be more sensitive in terms of iPL to LPA stenosis. Overall, Y-grafts show significantly higher resistance than LT/ECCs due in part to the intrinsic graft geometry, which calls for a better Y-graft design. 


\section{Conflict of Interest Statement}

M.A.F. received a research grant, modest, National Institutes of Health R01; is a consultant and on the advisory board, modest, for Edwards Life Sciences MRI Core Lab; and received other, modest, from AMAG FACT trial site and Cooley's Anemia Foundation MRI Core Lab. All other authors have nothing to disclose with regard to commercial support.

\section{References}

1. Fontan F, Baudet E. Surgical repair of tricuspid atresia. Thorax. 1971;26:240-8.

2. Pekkan K, Whited B, Kanter K, Sharma S, deZelicourt D, Sundareswaran K, et al. Patient-specific surgical planning and hemodynamic computational fluid dynamics optimization through free-form haptic anatomy editing tool (SURGEM). Med Biol Eng Comput. 2008;46:1139-52.

3. Sundareswaran KS, de Zélicourt D, Sharma S, Kanter K, Spray T, Rossignac J, et al. Correction of pulmonary arteriovenous malformation using image-based surgical planning. JACC Cardiovasc Imaging. 2009;2:1024-30.

4. Restrepo M, Luffel M, Sebring J, Kanter K, DelNido P, Veneziani A, et al. Surgical planning of the total cavopulmonary connection: robustness analysis. Ann Biomed Eng. 2014;43:1321-34.

5. Tang E, Restrepo M, Haggerty C, Mirabella L, Whitehead K, Bethel J, et al. A retrospective cohort of 100 Fontan connections: relationship between geometric features and hemodynamics outcomes. J Am Coll Cardiol. 2013;61:E490.

6. Haggerty CM, Restrepo M, Tang E, deZelicourt D, Sundareswaren K, Mirabella L, et al. Fontan hemodynamics from 100 patient-specific cardiac magnetic resonance studies: a computational fluid dynamics analysis. J Thorac Cardiovasc Surg. 2013;148:1-10.

7. Restrepo M, Mirabella L, Tang E, Haggerty C, Khiabani R, Fynn-Thompson F, et al. Fontan pathway growth: a quantitative evaluation of lateral tunnel and extracardiac cavopulmonary connections using serial cardiac magnetic resonance. Ann Thorac Surg. 2014;97:916-22.

8. Kogon B. Is the extracardiac conduit the preferred Fontan approach for patients with univentricular hearts? The extracardiac conduit is the preferred Fontan approach for patients with univentricular hearts. Circulation. 2012;126: 2511-5.

9. Khiabani RH, Restrepo M, Tang E, De Zelicourt D, Sotiropoulos F, Fogel M, et al. Effect of flow pulsatility on modeling the hemodynamics in the total cavopulmonary connection. J Biomech. 2012;45:2376-81.

10. Tang E, Restrepo M, Haggerty CM, Mirabella L, Bethel J, Whitehead K, et al. Geometric characterization of patient-specific total cavopulmonary connections and its relationship to hemodynamics. JACC Cardiovasc Imaging. 2014;7: 215-24.

11. Duncan BW, Desai S. Pulmonary arteriovenous malformations after cavopulmonary anastomosis. Ann Thorac Surg. 2003;76:1759-66.

12. Shah MJ, Rychik J, Fogel MA, Murphy JD, Jacobs ML. Pulmonary AV malformations after superior cavopulmonary connection: resolution after inclusion of hepatic veins in the pulmonary circulation. Ann Thorac Surg. 1997;63:960-3.

13. Kanter KR, Haggerty CM, Restrepo M, de Zelicourt D, Rossignac J, Parks J, et al. Preliminary clinical experience with a bifurcated Y-graft Fontan procedure - a feasibility study. J Thorac Cardiovasc Surg. 2012;144:383-9.
14. Haggerty CM, Kanter KR, Restrepo M, de Zelicourt D, Parks J, Rossignac J, et al. Simulating hemodynamics of the Fontan Y-graft based on patient-specific in vivo connections. J Thorac Cardiovasc Surg. 2013;145:663-70.

15. Yang W, Feinstein JA, Shadden SC, Vignon-Clementel IE, Marsden AL Optimization of a Y-graft design for improved hepatic flow distribution in the Fontan circulation. J Biomech Eng. 2013;135:011002.

16. Yang W, Chan FP, Reddy VM, Marsden AL, Feinstein JA. Flow simulations and validation for the first cohort of patients undergoing the Y-graft Fontan procedure. J Thorac Cardiovasc Surg. 2015;149:247-55.

17. Marsden AL, Bernstein AJ, Reddy VM, Shadden S, Spilker R, Chan F, et al. Evaluation of a novel Y-shaped extracardiac Fontan baffle using computational fluid dynamics. J Thorac Cardiovasc Surg. 2009;137:394-403.e2.

18. Yang W, Feinstein JA, Marsden AL. Constrained optimization of an idealized Y-shaped baffle for the Fontan surgery at rest and exercise. Comput Methods Appl Mech Eng. 2010;199:2135-49.

19. Dur O, DeGroff CG, Keller BB, Pekkan K. Optimization of inflow waveform phase-difference for minimized total cavopulmonary power loss. J Biomech Eng. 2010;132:031012.

20. Frakes DH, Smith MJ, Parks J, Sharma S, Fogel SM, Yoganathan AP. New techniques for the reconstruction of complex vascular anatomies from MRI images. J Cardiovasc Magn Reson. 2005;7:425-32.

21. Frakes DH, Conrad CP, Healy TM, Monaco J, Fogel M, Sharma S, et al. Application of an adaptive control grid interpolation technique to morphological vascular reconstruction. IEEE Trans Biomed Eng. 2003;50: 197-206.

22. Frakes D, Smith M, de Zelicourt D, Pekkan K, Yoganathan A. Three-dimensional velocity field reconstruction. J Biomech Eng. 2004;126:727.

23. Sundareswaran KS, Frakes DH, Fogel MA, Soerensen DD, Oshinski JN, Yoganathan AP. Optimum fuzzy filters for phase-contrast MRI segmentation. J Magn Reson Imaging. 2009;29:155-65.

24. De Zélicourt D, Ge L, Wang C, Sotiropoulos F, Gilmanov A, Yoganathan A. Flow simulations in arbitrarily complex cardiovascular anatomies-an unstructured Cartesian grid approach. Comput Fluids. 2009;38:1749-62.

25. Schiavazzi DE, Kung EO, Marsden AL, Baker C, Pennati G, Hsia T, et al. Hemodynamic effects of left pulmonary artery stenosis after superior cavopulmonary connection: a patient-specific multiscale modeling study. $J$ Thorac Cardiovasc Surg. 2015;149:689-96.e3.

26. Ghaferi AA, Hutchins GM. Progression of liver pathology in patients undergoing the Fontan procedure: chronic passive congestion, cardiac cirrhosis, hepatic adenoma, and hepatocellular carcinoma. J Thorac Cardiovasc Surg. 2005;129: 1348-52.

27. Asrani SK, Asrani NS, Freese DK, Phillips S, Warnes C, Heimbach J, et al. Congenital heart disease and the liver. Hepatology. 2012;56:1160-9.

28. Mertens L, Hagler DJ, Sauer U, Somerville J, Gewillig M. Protein-losing enteropathy after the Fontan operation: an international multicenter study. PLE study group. J Thorac Cardiovasc Surg. 1998;115:1063-73.

29. Wootton DM, Ku DN. Fluid mechanics of vascular systems, diseases, and thrombosis. Annu Rev Biomed Eng. 1999;1:299-329.

30. Rayz VL, Boussel L, Ge L, Leach J, Martin A, Lawton M, et al. Flow residence time and regions of intraluminal thrombus deposition in intracranial aneurysms. Ann Biomed Eng. 2010;38:3058-69.

Key Words: Fontan, Y-graft, hepatic flow distribution, single ventricle 